\title{
B Cell Depletion: Rituximab in Glomerular Disease and Transplantation
}

\author{
S. Marinaki C. Skalioti J.N. Boletis \\ Department of Nephrology and Renal Transplant Unit, General Hospital 'Laiko', Athens, Greece
}

\section{Key Words}

B cell depletion - Rituximab - Membranous nephropathy · Lupus nephritis · ANCA-associated vasculitis · Transplantation

\begin{abstract}
B cells play a central role in the pathogenesis of many autoimmune diseases. Selective targeting can be achieved with the use of the monoclonal antibody rituximab. In addition to being a drug for non-Hodgkin's lymphoma, rituximab is also an FDA-approved treatment for refractory rheumatoid arthritis and, since recently, ANCA vasculitis. It has shown efficacy in many autoimmune diseases. This review will discuss current evidence and the rationale of the use of rituximab in glomerular diseases, including randomized controlled trials. The focus will be on the use of rituximab in idiopathic membranous nephropathy, systemic lupus erythematosus and ANCA-associated vasculitis. The emerging role of rituximab in renal transplantation, where it seems to be important for the desensitization protocols for highly sensitized patients as well as for the preconditioning of $\mathrm{ABO}$-incompatible recipients and the treatment of antibody-mediated rejection, will also be addressed.

(c) 2013 S. Karger AG, Basel
\end{abstract}

\section{Introduction}

B cells occupy a central role in normal immunity. They interact with antigen-presenting cells, act as antigen-presenting cells themselves, provide co-stimulatory support to $\mathrm{T}$ cells, differ from plasma cells and produce antibodies. B cells have the ability to expand and clonally proliferate. As expected, abnormal B cell function plays a major role in immune dysregulation such as autoimmunity [1]. Furthermore, B cells are implicated in the pathogenesis of $\mathrm{T}$ cell autoreactivity. Abnormal B and T cell interactions may be amplified by T cell-derived cytokines such as the B lymphocyte stimulator and a proliferation-inducing ligand [2]. Additionally, B cells have been found in affected tissues from nasal biopsies of patients with ANCA-associated vasculitis (AASV) [3] or from renal biopsies of patients with membranous nephropathy [4]. 


\section{B Cell-Directed Therapies}

B cell-directed therapies include those that deplete B cells and those that alter B cell function. B cell depletion can be achieved by using monoclonal antibodies against B cellspecific antigens such as CD20, CD19 and CD22. Most monoclonal antibodies currently in use are against the anti-CD20 receptor. $B$ cell modulation comprises the blockade of the following cytokines: B cell-activating factor/B lymphocyte stimulator, proliferation-inducing ligand and their receptors, as well as co-stimulation blockade [2].

Rituximab is a mouse/human IgG1k chimeric monoclonal antibody against the CD20 cell surface receptor of the B cell. CD20 is expressed on immature, mature and activated B cells but not on long-lived plasma cells. Rituximab depletes B cells by three mechanisms: antibodydependent cell-mediated cytotoxicity, complement-dependent cytotoxicity and apoptosis. One course of rituximab effectively depletes B cells for 6-9 months in over $80 \%$ of the patients [5]. Rituximab has first been licensed for the treatment of non-Hodgkin's lymphoma in the 1990s and has been approved for the treatment of rheumatoid arthritis in 2006. Thereafter, it has been used increasingly in autoimmune diseases. At first, the standard dosing regimens were either the so-called lymphoma protocol (four weekly doses of $375 \mathrm{mg} / \mathrm{m}^{2}$ ) or the rheumatoid arthritis protocol (two doses of $1 \mathrm{~g}, 2$ weeks apart). With growing evidence of its use, many adaptations have been made, including modified shorter courses or prolongation of treatment at fixed-dose intervals [6]. Rituximab has been widely used and is considered a safe drug. Most adverse events include minor infusion reactions that only rarely limit its use. According to the literature, up to $8 \%$ of all lymphoma patients experience a delayed neutropenia that may be profound but finally resolves [7]. True estimates about the incidence of infections after rituximab treatment are difficult because most patients receive concomitant immunosuppression. Rituximab use is not associated with an increased risk neither for common nor for opportunistic infections [1]. Concern has been raised about the relation of rituximab with progressive multifocal leukoencephalopathy. However, no definite conclusion can be drawn, since such patients also received intensive immunosuppression. On the other hand, the syndrome occurred also in patients who had never received rituximab [8].

Since rituximab does not deplete long-lived plasma cells, there is no decrease in immunoglobulin levels. However, a slight decrease in IgG occurs after repeated dosing. Human antichimeric antibodies develop in $5-30 \%$ of the patients. They may shorten the time of effective $\mathrm{B}$ cell depletion, but the true incidence and importance of these antibodies are unknown [1].

Rituximab has been used in idiopathic and secondary glomerulonephritides and in renal transplantation. Specifically in idiopathic glomerulonephritides, most of the available data concern the use of rituximab in idiopathic membranous nephropathy (IMN) and steroidresistant nephrotic syndrome (NS). Secondary forms of glomerulonephritis include systemic lupus erythematosus (SLE) and AASV. In renal transplantation, rituximab has been used for the desensitization of highly sensitized patients before transplantation and as part of the preconditioning of the recipient before $\mathrm{ABO}$-incompatible transplantations. Furthermore, it is used in severe immune-mediated, antibody-associated rejection.

\section{Idiopathic Membranous Nephropathy}

Since IMN is characterized by autoantibody formation and immune complex deposition, from the pathogenetic point of view, the use of rituximab is reasonable. Ruggenenti et al. [9] were the first who used rituximab as first-line therapy in 8 patients with IMN. Sustained remission with maximal reduction of proteinuria by $66 \%$ occurred after 12 months. In the 
meantime, several case series and small prospective trials reporting that rituximab is efficacious in IMN have been published. A meta-analysis published in 2009 [10] summarized the results of rituximab use in 85 patients with IMN. It showed good remission rates $115-20 \%$ had complete and $35-40 \%$ had partial remission) and excellent tolerance. Our experience with 12 IMN patients who were treated with rituximab was sustained remission in 11 of these patients during a median follow-up of 48 months. Interestingly, remission first occurred after 6 months [11]. Rituximab also seems to be effective in recurrent membranous nephropathy after transplantation [12]. A comparison of the two main dosing regimens of rituximab for IMN therapy showed equal efficacy [13]. An interesting study by Ruggenenti et al. [14] evaluated the effect of rituximab on the morphofunctional abnormalities of IMN. After induction of remission, there was reabsorption of the subepithelial dense deposits in repeat biopsies, which strongly suggested interference of rituximab with the pathogenetic mechanism. In most reports, the drug was used as a first-line treatment. Despite its excellent tolerability and the good efficacy data, rituximab is not licensed for the treatment of membranous nephropathy, since data from randomized controlled trials are missing. We recommend its use only in selected patients who have contraindications in receiving 'conventional' immunosuppressive regimens.

\section{Refractory NS}

Most of the literature comes from pediatric experience because pediatricians are most often confronted with this disease, either in the form of steroid dependency and frequent relapses or as steroid-resistant NS. A study on 70 children treated with rituximab reported a response rate of $82 \%$ in steroid-dependent NS, $44 \%$ in steroid-resistant NS and $60 \%$ in recurrent posttransplant NS [15]. Another report on 57 patients showed sustained remission in $83 \%$ of the steroid-dependent NS cases and in $21 \%$ of the steroid-resistant NS cases at 12 months [16]. Both reports showed good results in cases in which rituximab was used as rescue therapy. Nevertheless, questions about how early or late rituximab should be administered, the duration of remission and the indications for retreatment are still open.

\section{ANCA-Associated Vasculitis}

Conventional immunosuppression has changed the fatal course of primary systemic AASV to a chronic disease with remission periods and relapses. However, therapy-related toxicity (especially infections), primary treatment failures in up to $20 \%$ and a relapse rate of $>50 \%$ at 5 years together with a persistently high mortality rate reflect the unmet needs in the standard of care of these patients [17]. The therapeutic limitations of standard immunosuppression and the accumulating evidence of the importance of ANCA in the pathogenesis of this disease constitute the rationale for rituximab use in AASV. From the standpoint of efficacy, rituximab is effective in AASV. In small case series and open-label trials, a clinical response occurred in the majority of patients, with the exception of those with refractory granulomatous manifestations $[6,18]$.

Recently, two randomized trials using rituximab as induction therapy in AASV have been published [19, 20]. In the European RITUXVAS trial [19], 44 patients were randomly assigned to receive corticosteroids plus either rituximab or cyclophosphamide as induction therapy for AASV with severe renal involvement. After 12 months, there was no difference, neither in remission rates ( 76 vs. $82 \%, p=0.68$ ) nor in the serious adverse event rates ( 42 vs. $36 \%$ ). Both treatment arms had an early mortality of $18 \%$. The RAVE-ITN trial [20], conducted in 
the USA, is a noninferiority trial directly comparing rituximab to oral cyclophosphamide as induction therapy in 197 patients with severe but not life-threatening AASV. Rituximab was not inferior to cyclophosphamide for induction of remission at 6 months and it even showed superiority in patients with frequent relapses. Adverse event rates, however, were comparable. In both trials, rituximab showed no superiority in terms of safety, but both were shortterm trials and therefore not designed to detect possible advantages of cyclophosphamide avoidance translating into lower long-term mortality.

Regarding rituximab use as maintenance therapy, although encouraging, there are no conclusive data about medium- and long-term efficacy and safety. Open-label trials with multiple dosing regimens have been conducted [6]. To our knowledge, the only randomized trial that evaluated rituximab for maintenance of remission is the RAVE-ITN trial [21]. Induction therapy with rituximab was followed by placebo, whereas cyclophosphamide therapy was followed by azathioprine after 3-6 months. A single course of rituximab was as effective as conventional therapeutic regimens in remission maintenance at 12 and 18 months. Especially in frequently relapsing disease, rituximab continued to be superior to conventional immunosuppression at 12 months but not at 18 months when B cells had reappeared. The relapse rates did not differ significantly between the two groups (32 vs. 29\%). The safety of the two therapeutic regimens was similar, as was patient survival during the 18 months of follow-up. Questions about the treatment of relapses preemptively or after the relapse and about the use of rituximab at fixed-dose intervals or with and without the concomitant use of steroids are still open. Nevertheless, rituximab seems to be a promising way to avoid long-term toxicity without losing the high-efficacy benefit that is gained with cytotoxic drugs.

\section{Lupus Nephritis}

SLE is the prototypic autoimmune disease, with a variety of autoantibodies directed against diverse cellular components and multiorgan involvement. Since B cells are a key component of the dysregulated autoimmune response, there is a rationale for the wide use of B cell-depleting agents in this disease. Regarding SLE nephritis, rituximab has been used as induction therapy in refractory cases or in conjunction with low-dose conventional immunosuppression. Cumulative evidence from small, open-label trials suggests that rituximab is both safe and effective in lupus nephritis [6,22].

A multicenter phase III trial [23] of rituximab versus placebo given as induction therapy in addition to mycophenolate mofetil (MMF) and high-dose steroids in both treatment arms did not attain its primary end point (renal response) in 144 SLE nephritis patients at 52 weeks. This study disappointed investigators worldwide. One explanation for favoring rituximab seems logical: the trial was intended to detect a large clinical effect in patients with very active disease. This was not possible for a biological agent given in addition to high-dose conventional therapy in both treatment arms. Further studies using rituximab in targeted populations are needed. For the use of rituximab as maintenance therapy, data from small, uncontrolled studies showed that sustained B cell depletion with repeated courses of rituximab either in conjunction with low doses of MMF/steroids or as monotherapy is effective and helps avoid long-term cyclophosphamide toxicity [6].

From our experience, the addition of rituximab to MMF is effective for the treatment of relapsing proliferative lupus nephritis [22].

Recent recommendations suggest the use of rituximab either in addition to mycophenolic acid or to cyclophosphamide in refractory cases of proliferative lupus nephritis [24]. 


\section{Renal Transplantation}

Over the past 2 decades, immunosuppressive strategies in kidney transplantation have focused on targeting T cells. However, there is increasing interest in the role of B cells, plasma cells and autoantibodies in transplantation. The use of rituximab first emerged after its use in ABO-incompatible renal transplantation, where it successfully replaced the more invasive splenectomy as part of the recipient-preconditioning protocol [25]. Rituximab has also shown efficacy as part of desensitization protocols for highly sensitized patients [26]. Its use in acute antibody-mediated rejection, in mixed cellular and humoral as well as cellular rejection resistant to $\mathrm{T}$ cell therapies amounts to a total of 150 patients [27]. The role of anti-CD20 remains to be better determined. From the pathogenetic point of view, it effectively depletes circulating and intragraft-naïve B cells, plasmablasts and short-lived plasma cells but not long-lived, antibody-producing plasma cells, and it mainly acts via inhibition of $\mathrm{T}$ cell activation and by preventing the generation of new, alloantibody-producing B cells after repopulation. From the clinical point of view, it seems partially effective in resolving acute antibodymediated rejection, but always in conjunction with other therapies such as IV immunoglobulin and/or plasmapheresis. Anti-CD20 does not seem to be effective in chronic antibodymediated rejection, where newer, plasma cell-depleting agents such as the proteasome inhibitor bortezomib show greater benefit [27]. One major concern about the use of B celldepleting agents in transplantation is that they also have inhibitory capacity, thereby prohibiting the induction of tolerance [27].

Future studies on B cell depletion should provide more insight into the role and interplay of the different $B$ cell subsets before and after depletion and use combinations of newer agents to achieve not only depletion but more sophisticated B cell modulation.

\section{References}

1 Walsh M, Jayne DRW: Targeting the B cell in vasculitis. Pediatr Nephrol 2009;24:1267-1275.

12 Doerner T, Burmester GR: New approaches of B-cell-directed therapy: beyond rituximab. Curr Opin Rheumatol 2008;20:263-268.

-3 Popa ER, Franssen CF, Limberg PC, et al: In vitro cytokine production and proliferation of T cells from patients with anti-proteinase- 3 and antimyeloperoxidase-associated vasculitis, in response to proteinase- 3 and myeloperoxidase. Arthritis Rheum 2002;46:1894-1904.

4 Cohen CD, Calvaresi N, Armelloni S, et al: CD20-positive infiltrates in human membranous glomerulonephritis. J Nephrol 2005; 18:328-333.

5 Anolik JH, Campbell D, Felgar RE, et al: The relationship of the FcgammaRIIIa genotype to degree of B cell depletion by rituximab in the treatment of systemic lupus erythematosus. Arthritis Rheum 2003;48:455-459.

6 Smith KG, Jones RB, Burns SM, Jayne DR: Long-term comparison of rituximab treatment for refractory systemic lupus erythematosus and vasculitis: remission, relapse and re-treatment. Arthritis Rheum 2006;54:29702982.

7 Chaiwatanatorn K, Lee N, Grigg A, et al: Delayed-onset neutropenia associated with rituximab therapy. Br J Haematol 2003;121:913-918.

-8 Harris HE: Progressive multifocal leucoencephalopathy in a patient with systemic lupus erythematosus treated with rituximab. Rheumatology 2008;47:224-225.

$>9$ Ruggenenti P, Chiurchiu C, Brusegan V, et al: Rituximab in idiopathic membranous nephropathy: a one-year prospective study. J Am Soc Nephrol 2003;14:1851-1857.

$\checkmark 10$ Bomback AS, Derebail VK, McGregor JG, et al: Rituximab therapy for membranous nephropathy: a systematic review. Clin J Am Soc Nephrol 2009;4:734-744.

11 Lionaki S, Marinaki S, Nakopoulou L, et al: Depletion of B lymphocytes in idiopathic membranous glomerulopathy: results from patients with extended follow-up. Nephron Extra 2013;3:1-11.

12 El-Zoghby ZM, Grande JP, Fraile MG, et al: Recurrent idiopathic membranous nephropathy: early diagnosis by protocol biopsies and treatment with anti-CD20 monoclonal antibodies. Am J Transplant 2009;9:2800-2807.

13 Fervenza FC, Abraham RS, Erickson SB, et al: Rituximab therapy in idiopathic membranous nephropathy: a 2-year study. Clin J Am Soc Nephrol 2010;5:2188-2198.

14 Ruggenenti P, Cravedi P, Sghirlanzoni MC, et al: Effects of rituximab on morphofunctional abnormalities of membranous glomerulopathy. Clin J Am Soc Nephrol 2008;3:1652-1659. 
15 Prytula A, Iijima K, Kamei K, et al: Rituximab in refractory nephrotic syndrome. Pediatr Nephrol 2010;25: 461-468.

16 Gulati A, Sinha A, Jordan SC, et al: Efficacy and safety of treatment with rituximab for difficult steroid-resistant and -dependent nephrotic syndrome: multicenter report. Clin J Am Soc Nephrol 2010;5:2207-2212.

17 Booth AD, Pusey CD, Jayne DR: Renal vasculitis - an update in 2004. Nephrol Dial Transplant 2004;19:19641968.

18 Aries PM, Hellmich B, Voswinkel J, et al: Lack of efficacy of rituximab in Wegener's granulomatosis with refractory granulomatous manifestations. Ann Rheum Dis 2006;65:853-858.

-19 Jones RB, Tervaert JW, Hauser T, et al, European Vasculitis Study Group: Rituximab versus cyclophosphamide in ANCA-associated renal vasculitis. N Engl J Med 2010;363:211-220.

20 Stone JH, Merkel PA, Spiera R, et al, RAVE-ITN Research Group: Rituximab versus cyclophosphamide for ANCA-associated vasculitis. N Engl J Med 2010;363:221-232.

21 Specks U, Merkel PA, Seo P, et al, RAVE-ITN Research Group: Efficacy of remission-induction regimens for ANCA-associated vasculitis. N Engl J Med 2013;369:417-427.

22 Boletis JN, Marinaki S, Skalioti C, et al: Rituximab and mycophenolate mofetil for relapsing proliferative lupus nephritis: a long-term prospective study. Nephrol Dial Transplant 2009;24:2157-2160.

23 Rovin BH, Furie R, Latinis K, et al, LUNAR Investigator Group: Efficacy and safety of rituximab in patients with active proliferative lupus nephritis: the Lupus Nephritis Assessment with Rituximab study. Arthritis Rheum 2012;64:1215-1226.

24 Bertsias GK, Tektonidou M, Amoura Z, et al, European League Against Rheumatism and European Renal Association-European Dialysis and Transplant Association: Joint European League Against Rheumatism and European Renal Association-European Dialysis and Transplant Association (EULAR/ERA-EDTA) recommendations for the management of adult and paediatric lupus nephritis. Ann Rheum Dis 2012;71:1771-1782.

-25 Tydén G, Kumlien G, Genberg H, et al: ABO incompatible kidney transplantations without splenectomy, using antigen-specific immunoadsorption and rituximab. Am J Transplant 2005;5:145-148.

26 Vo AA, Lukovsky M, Toyoda M, et al: Rituximab and intravenous immune globulin for desensitization during renal transplantation. N Engl J Med 2008;359:242-251.

27 Zarkhin V, Chalasani G, Sarwal MM: The yin and yang of B cells in graft rejection and tolerance. Transplant Rev $2010 ; 24: 67-78$. 